\title{
Enhancer decommissioning by LSD1 during embryonic stem cell differentiation
}

\author{
Warren A. Whyte ${ }^{1,2,{ }^{*}}$, Steve Bilodeau ${ }^{1,{ }^{*}}$, David A. Orlando ${ }^{1}$, Heather A. Hoke ${ }^{1,2}$, Garrett M. \\ Frampton $^{1,2}$, Charles T. Foster ${ }^{3,4}$, Shaun M. Cowley ${ }^{4}$, and Richard A. Young ${ }^{1,2}$ \\ ${ }^{1}$ Whitehead Institute for Biomedical Research, 9 Cambridge Center, Cambridge, Massachusetts \\ 02142, USA \\ 2Department of Biology, Massachusetts Institute of Technology, Cambridge, Massachusetts \\ 02139, USA \\ ${ }^{3}$ Department of Molecular Biology, Adolf-Butenandt Institut, Ludwig-Maximilians-Universität \\ München, 80336 Munich, Germany \\ ${ }^{4}$ Department of Biochemistry, University of Leicester, Leicester LE1 9HN, UK
}

\begin{abstract}
Transcription factors and chromatin modifiers are important in the programming and reprogramming of cellular states during development ${ }^{1,2}$. Transcription factors bind to enhancer elements and recruit coactivators and chromatin-modifying enzymes to facilitate transcription initiation $^{3,4}$. During differentiation a subset of these enhancers must be silenced, but the mechanisms underlying enhancer silencing are poorly understood. Here we show that the histone demethylase lysine-specific demethylase 1 (LSD1; ref. 5), which demethylates histone $\mathrm{H} 3$ on Lys 4 or Lys $9(\mathrm{H} 3 \mathrm{~K} 4 / \mathrm{K} 9)$, is essential in decommissioning enhancers during the differentiation of mouse embryonic stem cells (ESCs). LSD1 occupies enhancers of active genes that are critical for control of the state of ESCs. However, LSD1 is not essential for the maintenance of ESC identity. Instead, ESCs lacking LSD1 activity fail to differentiate fully, and ESC-specific enhancers fail to undergo the histone demethylation events associated with differentiation. At active enhancers, LSD1 is a component of the NuRD (nucleosome remodelling and histone deacetylase) complex, which contains additional subunits that are necessary for ESC differentiation. We propose that the LSD1-NuRD complex decommissions enhancers of the pluripotency program during differentiation, which is essential for the complete shutdown of the ESC gene expression program and the transition to new cell states.
\end{abstract}

\footnotetext{
Correspondence and requests for materials should be addressed to R.A.Y. (young@wi.mit.edu).

These authors contributed equally to this work.

Supplementary Information is linked to the online version of the paper at www.nature.com/nature.

Author Contributions W.A.W., S.B., H.A.H., C.T.F., S.M.C and R.A.Y. designed, conducted and interpreted the ChIP-Seq, immunofluorescence and expression experiments. W.A.W., D.A.O. and G.M.F. performed data analysis. The manuscript was written by S.B., W.A.W. D.A.O., H.A.H., G.M.F. and R.A.Y.

ChIP-Seq and GeneChip expression data are deposited in the Gene Expression Omnibus under accession number GSE27844.

Reprints and permissions information is available at www.nature.com/reprints.

The authors declare no competing financial interests.

Readers are welcome to comment on the online version of this article at www.nature.com/nature.
} 
The histone H3K4/K9 demethylase LSD1 (also known as KDM1A) is one of the chromatin regulators that have been implicated in the control of early embryogenesis ${ }^{6-8}$. Loss of LSD1 leads to embryonic lethality, and ESCs lacking LSD1 function fail to differentiate into embryoid bodies $^{6-8}$. These results suggest that LSD1 contributes to changes in chromatin that are critical to the differentiation of ESCs, but the role of LSD1 in this process is not yet understood. To investigate the function of LSD1 in ESCs, we first identified the sites it occupies in the genome by using chromatin immunoprecipitation coupled with massively parallel DNA sequencing (ChIP-Seq; Fig. 1 and Supplementary Fig. 1). The results revealed that LSD1 occupies the enhancers and core promoters of a substantial population of actively transcribed and bivalent genes (Fig. 1a, b and Supplementary Table 1). Inspection of individual gene tracks showed that LSD1 occupies well-characterized enhancer regions together with the ESC master transcription factors Oct4, Sox2 and Nanog and the Mediator coactivator (Fig. 1b and Supplementary Fig. 1). Loci bound by Oct4, Sox2 and Nanog are generally associated with Mediator and p300 coactivators and have enhancer activity ${ }^{9,10}$. A global view of enhancer regions occupied by Oct4, Sox2, Nanog and Mediator confirmed that $97 \%$ of the 3,838 high-confidence enhancers were also occupied by LSD1 $\left(P<10^{-9}\right)$ (Fig. 1c and Supplementary Table 2). This is consistent with evidence that LSD1 can interact with Oct4 (refs 11, 12). LSD1 signals were also observed at core promoter regions with RNA polymerase II (Pol II) and TATA-binding protein (TBP; Fig. 1d). The density of LSD1 signals at enhancers was higher than at core promoters $\left(P<10^{-16}\right.$; Supplementary Fig. 1), indicating that LSD1 is associated predominantly with the enhancers of actively transcribed genes in ESCs.

It was striking to find that LSD1 is associated with active genes in ESCs because previous studies have shown that LSD1 is not essential for the maintenance of ESC state but is required for normal differentiation ${ }^{6-8}$. We used an ESC differentiation assay to further investigate the involvement of LSD1 in cell state transitions (Fig. 2a, b). Prolonged depletion of Oct4 in ZHBTc4 ESCs with doxycycline causes loss of pluripotency and differentiation into trophectoderm ${ }^{13}$. As expected, loss of Oct 4 expression led to a rapid loss of ESC morphology and a marked decrease in the levels of SSEA-1 and alkaline phosphatase, two markers of ESCs (Fig. 2c and Supplementary Fig. 2). When these ESCs were treated with the LSD1 inhibitor tranylcypromine (TCP) during Oct4 depletion, they failed to undergo the morphological changes associated with differentiation of ESCs (Fig. 2c). Instead, the TCP-treated cells formed small colonies resembling those of untreated ESCs and maintained expression of SSEA-1 and alkaline phosphatase (Fig. 2c and Supplementary Fig. 3). Very similar results were partly obtained in LSD1 knockout ESCs (Supplementary Figs 4 and 5) and in cells treated with another LSD1 inhibitor, pargyline, or a short hairpin RNA against LSD1 (Supplementary Figs 2 and 3). LSD1 inhibition also caused an increase in cell death during differentiation, as has been observed with cells lacking LSD1 in other assays ${ }^{7,8}$. These results suggest that LSD1 may be required for ESCs to silence the ESC gene expression program completely.

Further analysis of ESCs that were forced to differentiate in the absence of LSD1 activity confirmed that these cells failed to make a complete transition from the ESC gene expression program; although key genes of the trophectoderm gene expression program 
were activated, including $C d x 2$ and $E s x l$ (ref. 14), there was incomplete repression of many ESC genes, including Sox2 and Fbox15 (Fig. 2d). A global analysis confirmed that a set of genes neighbouring LSD1-occupied enhancers in ESCs are repressed during differentiation and that the repression of this set of genes is partly relieved in the presence of TCP (Fig. 2e and Supplementary Table 3). Similar results were obtained with LSD1 knockout cells (Supplementary Figs 4 and 5) and with cells treated with either pargyline or a short hairpin RNA against LSD1 (Supplementary Fig. 3). These results indicate that the trophectoderm differentiation program can be induced in cells lacking LSD1 function, but the ESC program is not fully silenced in these cells.

To gain further insight into the role of LSD1 in ESC differentiation, we investigated whether LSD1 is associated with previously described complexes, including NuRD, cofactor of REST (coREST), and the androgen receptor/oestrogen receptor complexes ${ }^{8,15-17}$. We first studied whether the LSD1 found at Oct4-occupied genes is a component of NuRD, because Oct4 and Nanog have been reported to interact with several components of NuRD ${ }^{11,12,18}$. ChIP-Seq experiments confirmed that NuRD subunits Mi-2 $\beta$, HDAC1 and HDAC2 together occupy sites with LSD1 at enhancers $\left(P<10^{-9}\right.$; Fig. 3 and Supplementary Table 1). Immunoprecipitation of LSD1 confirmed its association with Mi-2 $\beta$, HDAC1 and HDAC2 (Fig. 3b, c). We then investigated whether LSD1 is associated with CoREST; ChIP-Seq data revealed that a minor fraction of LSD1 occupies sites together with CoREST and REST (2\% and 6\%, respectively) (Supplementary Fig. 6 and Supplementary Table 1). As expected, LSD1-REST sites were frequently found associated with neuronal genes (Supplementary Fig. 7 and Supplementary Table 4). Immunoprecipitation experiments confirmed that LSD1 is associated with CoREST (Fig. 3b, c). Androgen receptor and oestrogen receptor are not expressed in ESCs, as indicated by the lack of histone H3K79me2 and H3K36me3 (modifications associated with transcriptional elongation) at the genes encoding these proteins (Supplementary Table 1). Further examination of the ChIP-Seq data revealed that enhancers were significantly more likely to be occupied by the LSD1 and NuRD proteins than by REST and CoREST $\left(P<10^{-9}\right)$ (Fig. 3d and Supplementary Fig. 8). Multiple components of NuRD are dispensable for ESC state but are required for normal differentiation ${ }^{6,19-21}$. ESCs with decreased levels of the core NuRD ATPase Mi-2 $\beta$ failed to differentiate properly and partly maintained expression of SSEA-1, alkaline phosphatase and ESC genes (Supplementary Fig. 9), which are the same phenotypes as those we observed with decreased levels of LSD1. These results indicate that LSD1 at enhancers is associated with a NuRD complex that is essential for normal cell state transitions.

Nucleosomes with histone H3K4me1 are commonly found at enhancers of active genes and are a substrate for LSD1 (refs 5, 22). If LSD1-dependent H3K4me1 demethylase activity is involved in enhancer silencing during ESC differentiation, LSD1 inhibition should cause the retention of $\mathrm{H} 3 \mathrm{~K} 4 \mathrm{me} 1$ levels at active ESC enhancers when differentiation is induced. During trophectoderm differentiation with control ESCs, we found decreased levels of p300 and $\mathrm{H} 3 \mathrm{~K} 27 \mathrm{ac}$ at a set of active ESC enhancers, suggesting that these enhancers were being silenced (Supplementary Fig. 10). The levels of H3K4me1 at enhancers were also decreased, as seen for example at Leftyl (Fig. 4a and Supplementary Table 5), whereas the levels of H3K4me1 increased at newly active trophectoderm genes such as Gata2 (Fig. 4b). In 
contrast, H3K4me1 signals were higher at LSD1-occupied enhancers in differentiating ESCs treated with TCP than in control cells, including Leftyl and Sox2 (Fig. 4a, c). Most enhancers $(1,722$ of 2,755$)$ that were occupied by LSD1 and that experienced decreased levels of $\mathrm{H} 3 \mathrm{~K} 4 \mathrm{me} 1$ during differentiation retained $\mathrm{H} 3 \mathrm{~K} 4 \mathrm{me} 1$ in TCP-treated ESCs, in contrast to untreated control differentiating ESCs (Fig. 4d, e). These results are consistent with the model that LSD1 demethylates H3K4me1 at the enhancers of ESC-specific genes during differentiation and that this activity is essential to fully repress the genes associated with these enhancers.

Our results indicate that an LSD1-NuRD complex is required for silencing of ESC enhancers during differentiation, which is essential for complete shutdown of the ESC gene expression program and the transition to new cell states. These results, together with those of previous studies on NuRD function ${ }^{18,21,23,24}$, suggest the following model for LSD1NuRD in enhancer decommissioning. LSD1-NuRD complexes occupy Oct4-regulated active enhancers in ESCs but do not substantially demethylate histone $\mathrm{H} 3 \mathrm{~K} 4$ because the H3K4 demethylase activity of LSD1 is inhibited in the presence of acetylated histones ${ }^{23,24}$. Enhancers occupied by Oct4, Sox 2 and Nanog are also occupied by the HAT p300 and nucleosomes with acetylated histones (Supplementary Fig. 10) ${ }^{10}$. Thus, as long as the enhancer-bound transcription factors recruit HATs to enhancers, the net effect of having both HATs and NuRD-associated HDACs present is to have sufficient levels of acetylated histones to suppress LSD1 demethylase activity. During ESC differentiation, the levels of Oct 4 and p300 are decreased, thus decreasing the level of acetylated histones, which in turn permits the demethylation of H3K4 by LSD1. Consistent with this model, we find that the shutdown of Oct4 leads to decreased levels of p300 and histone H3K27ac at enhancers that are occupied by Oct4 and LSD1 (Supplementary Figs 10 and 11), and this is coincident with decreased levels of methylated H3K4 (Fig. 4 and Supplementary Figs 12 and 13). This model would explain why key components of LSD1-NuRD complexes are not essential for the maintenance of ESC state but are essential for normal differentiation, when the active enhancers must be silenced. Additional HATs expressed in ESCs may also contribute to the dynamic balance of nucleosome acetylation. Future biochemical analysis of HAT, HDAC and demethylase complexes at enhancers will be valuable for testing this model and for further understanding how enhancers are regulated during differentiation.

We conclude that LSD1-NuRD complexes present at active promoters in ESCs are essential for normal differentiation, when the active enhancers must be silenced. Given that there is evidence that LSD1 is required for differentiation of multiple cell types ${ }^{6,25,26}$, LSD1 is likely to be generally involved in enhancer silencing during differentiation. The ESC gene expression program can be maintained in the absence of many other chromatin regulators ${ }^{2}$, and it is possible that some of these also have key functions in the transition from one transcriptional program to another during differentiation.

\section{METHODS SUMMARY}

\section{ESC culture conditions}

ESCs were grown on irradiated murine embryonic fibroblasts (MEFs) and passaged as described previously ${ }^{9}$. In drug treatment experiments, ESCs were split off MEFs and treated 
with $1 \mathrm{mM}$ TCP or $3 \mathrm{mM}$ pargyline to inhibit LSD1 activity. Lentiviral constructs were purchased from Open Biosystems and produced according to the Trans-lentiviral shRNA Packaging System (catalogue no. TLP4614).

\section{Differentiation assay, immunofluorescence, and alkaline phosphatase staining}

ZHBTc4 ESCs were split off MEFs in ESC medium containing $2 \mu \mathrm{g} \mathrm{ml}^{-1}$ doxycycline to decrease Oct4 expression levels. For immunofluorescence, ESCs were crosslinked, blocked and permeabilized before incubation with anti-Oct4 (Santa Cruz, sc-9081x; 1:200 dilution) or anti-SSEA1 (mc-480, Developmental Studies Hybridoma Bank; 1:20 dilution) antibodies. Alexa-conjugated secondary antibodies were used for detection. Staining of ESCs for alkaline phosphatase was achieved with the Alkaline Phosphatase Detection Kit (Millipore, SCR004). Cells were harvested at indicated time points for ChIP-Seq, quantitative polymerase chain reaction or expression array analyses.

\section{ChIP-Seq}

Chromatin immunoprecipitations (ChIPs) were performed and analysed as described previously ${ }^{9}$. The following antibodies were used: anti-LSD1 (Abcam, ab17721), anti-Mi-2b (Abcam, ab72418), anti-HDAC1 (Abcam, ab7028), anti-HDAC2 (Abcam, ab7029), antiREST (Millipore, 07-579), anti-CoREST (Abcam, ab32631), anti-H3K4me1 (Abcam, ab8895), anti-p300 (Santa-Cruz, sc-584) and anti-H3K27Ac (Abcam, ab4729).

For ChIP-Seq analyses, reads were aligned with Bowtie and analysed as described in Supplementary Information.

\section{Supplementary Material}

Refer to Web version on PubMed Central for supplementary material.

\section{Acknowledgments}

We thank J. Lovén, M. H. Kagey, J. Dowen, A. C. Mullen, A. Sigova, P. B. Rahl, T. Lee and members of Y. Shi's laboratory for experimental assistance, reagents and helpful discussions; and J.-A. Kwon, V. Dhanapal, J. Love, S. Gupta, T. Volkert, W. Salmon and N. Watson for assistance with ChIP-Seq, expression arrays and immunofluorescence imaging acquisition. This work was supported by a Canadian Institutes of Health Research Fellowship (S.B.), a Career Development Award from the Medical Research Council (S.M.C.), and by National Institutes of Health grants HG002668 and NS055923 (R.Y.).

\section{References}

1. Graf T, Enver T. Forcing cells to change lineages. Nature. 2009; 462:587-594. [PubMed: 19956253]

2. Young RA. Control of the embryonic stem cell state. Cell. 2011; 144:940-954. [PubMed: 21414485]

3. Fuda NJ, Ardehali MB, Lis JT. Defining mechanisms that regulate RNA polymerase II transcription in vivo. Nature. 2009; 461:186-192. [PubMed: 19741698]

4. Li B, Carey M, Workman JL. The role of chromatin during transcription. Cell. 2007; 128:707-719. [PubMed: 17320508]

5. Shi Y, et al. Histone demethylation mediated by the nuclear amine oxidase homolog LSD1. Cell. 2004; 119:941-953. [PubMed: 15620353] 
6. Wang J, et al. Opposing LSD1 complexes function in developmental gene activation and repression programmes. Nature. 2007; 446:882-887. [PubMed: 17392792]

7. Wang $\mathrm{J}$, et al. The lysine demethylase LSD1 (KDM1) is required for maintenance of global DNA methylation. Nature Genet. 2009; 41:125-129. [PubMed: 19098913]

8. Foster CT, et al. Lysine-specific demethylase 1 regulates the embryonic transcriptome and CoREST stability. Mol Cell Biol. 2010; 30:4851-4863. [PubMed: 20713442]

9. Kagey MH, et al. Mediator and cohesin connect gene expression and chromatin architecture. Nature. 2010; 467:430-435. [PubMed: 20720539]

10. Chen X, et al. Integration of external signaling pathways with the core transcriptional network in embryonic stem cells. Cell. 2008; 133:1106-1117. [PubMed: 18555785]

11. Pardo M, et al. An expanded Oct4 interaction network: implications for stem cell biology, development, and disease. Cell Stem Cell. 2010; 6:382-395. [PubMed: 20362542]

12. van den Berg DL, et al. An Oct4-centered protein interaction network in embryonic stem cells. Cell Stem Cell. 2010; 6:369-381. [PubMed: 20362541]

13. Niwa H, Miyazaki J, Smith AG. Quantitative expression of Oct-3/4 defines differentiation, dedifferentiation or self-renewal of ES cells. Nature Genet. 2000; 24:372-376. [PubMed: 10742100]

14. Rossant J, Cross JC. Placental development: lessons from mouse mutants. Nature Rev Genet. 2001; 2:538-548. [PubMed: 11433360]

15. Metzger E, et al. LSD1 demethylates repressive histone marks to promote androgen-receptordependent transcription. Nature. 2005; 437:436-439. [PubMed: 16079795]

16. Shi YJ, et al. Regulation of LSD1 histone demethylase activity by its associated factors. Mol Cell. 2005; 19:857-864. [PubMed: 16140033]

17. Wang Y, et al. LSD1 is a subunit of the NuRD complex and targets the metastasis programs in breast cancer. Cell. 2009; 138:660-672. [PubMed: 19703393]

18. Liang J, et al. Nanog and Oct4 associate with unique transcriptional repression complexes in embryonic stem cells. Nature Cell Biol. 2008; 10:731-739. [PubMed: 18454139]

19. Dovey OM, Foster CT, Cowley SM. Histone deacetylase 1 (HDAC1), but not HDAC2, controls embryonic stem cell differentiation. Proc Natl Acad Sci USA. 2010; 107:8242-8247. [PubMed: 20404188]

20. Kaji K, et al. The NuRD component Mbd3 is required for pluripotency of embryonic stem cells. Nature Cell Biol. 2006; 8:285-292. [PubMed: 16462733]

21. Scimone ML, Meisel J, Reddien PW. The Mi-2-like Smed-CHD4 gene is required for stem cell differentiation in the planarian Schmidtea mediterranea. Development. 2010; 137:1231-1241. [PubMed: 20223763]

22. Heintzman ND, et al. Distinct and predictive chromatin signatures of transcriptional promoters and enhancers in the human genome. Nature Genet. 2007; 39:311-318. [PubMed: 17277777]

23. Forneris F, Binda C, Vanoni MA, Battaglioli E, Mattevi A. Human histone demethylase LSD1 reads the histone code. J Biol Chem. 2005; 280:41360-41365. [PubMed: 16223729]

24. Lee MG, et al. Functional interplay between histone demethylase and deacetylase enzymes. Mol Cell Biol. 2006; 26:6395-6402. [PubMed: 16914725]

25. Musri MM, et al. Histone demethylase LSD1 regulates adipogenesis. J Biol Chem. 2010; 285:30034-30041. [PubMed: 20656681]

26. Choi J, et al. Histone demethylase LSD1 is required to induce skeletal muscle differentiation by regulating myogenic factors. Biochem Biophys Res Commun. 2010; 401:327-332. [PubMed: 20833138]

27. Okumura-Nakanishi S, Saito M, Niwa H, Ishikawa F. Oct-3/4 and Sox2 regulate Oct-3/4 gene in embryonic stem cells. J Biol Chem. 2005; 280:5307-5317. [PubMed: 15557334]

28. Yeom YI, et al. Germline regulatory element of Oct-4 specific for the totipotent cycle of embryonal cells. Development. 1996; 122:881-894. [PubMed: 8631266]

29. Nakatake Y, et al. Klf4 cooperates with Oct3/4 and Sox 2 to activate the Leftyl core promoter in embryonic stem cells. Mol Cell Biol. 2006; 26:7772-7782. [PubMed: 16954384] 
30. Ray S, et al. Context-dependent function of regulatory elements and a switch in chromatin occupancy between GATA 3 and GATA2 regulate Gata2 transcription during trophoblast differentiation. J Biol Chem. 2009; 284:4978-4988. [PubMed: 19106099] 

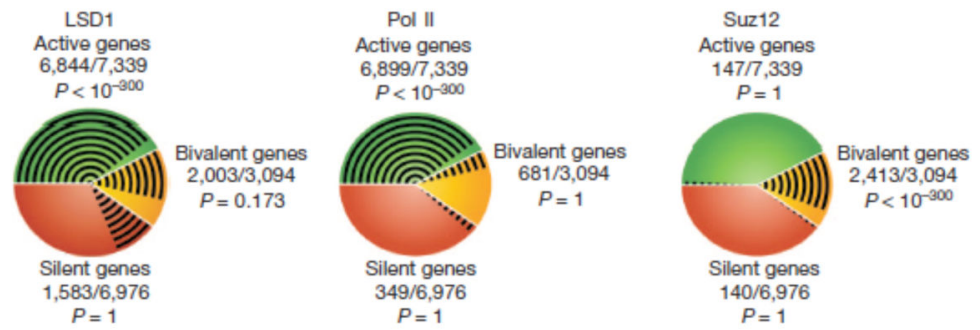

b
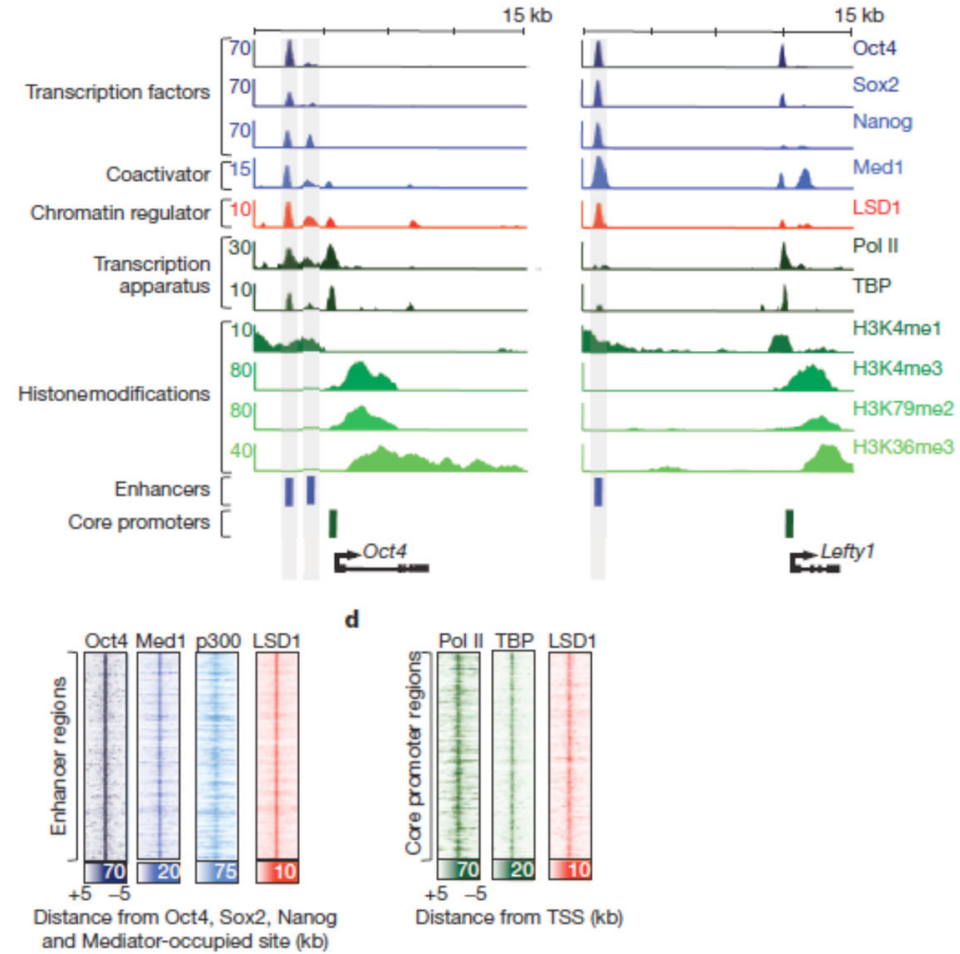

Figure 1. LSD1 is associated with enhancer and core promoter regions of active genes in ESCs a, LSD1 occupies a substantial population of actively transcribed genes in murine ESCs. The pie charts depict active (green), bivalent (yellow) and silent (red) genes, and the proportion (black lines) occupied by LSD1, Pol II or the Polycomb protein Suz12 (Supplementary Table 1 and Supplementary Information). The numbers of genes bound and the total number of genes in each of the active, bivalent and silent classes are shown. LSD1 ChIP-Seq data are from combined biological replicates using an antibody specific for LSD1 as determined by knockdown experiments (Supplementary Fig. 1). The $P$ value for each category was determined by a hypergeometric test. b, LSD1 occupies enhancers and core promoter regions of actively transcribed genes. Shown are ChIP-Seq binding profiles (reads per million) for ESC transcription factors (Oct4, Sox2, Nanog), coactivator (Med1), chromatin regulator (LSD1), the transcriptional apparatus (Pol II, TBP) and histone modifications (H3K4me1, H3K4me3, H3K79me2, H3K36me3) at the Oct4 (Pou5f1) and Leftyl loci in ESCs, with the $y$-axis floor set to 1. Gene models and previously described enhancer regions ${ }^{27-29}$ are shown below the binding profiles. c, LSD1 occupies enhancer sites. A density map is shown of ChIP-Seq data at Oct4, Sox2, Nanog and Med1 co- 
occupied enhancer regions. Data are shown for an ESC transcription factor (Oct4), coactivators (Med1 and p300) and a chromatin regulator (LSD1) in ESCs. Enhancers were defined as Oct4, Sox2, Nanog and Mediator co-occupied regions. More than $96 \%$ of the 3,838 high-confidence enhancers were co-occupied by LSD1 $\left(P<10^{-9}\right)$. Colour scale indicates ChIP-seq signal in reads per million. d, LSD1 occupies core promoter sites. Shown is a density map of ChIP-Seq data at transcriptional start sites (TSSs) of genes neighbouring the 3,838 previously defined enhancers (c). Data are shown for components of the transcription apparatus (Pol II and TBP) and the chromatin regulator LSD1 in ESCs. Core promoters were defined as the closest TSS from each enhancer. Colour scale indicates ChIPSeq signal in reads per million. 


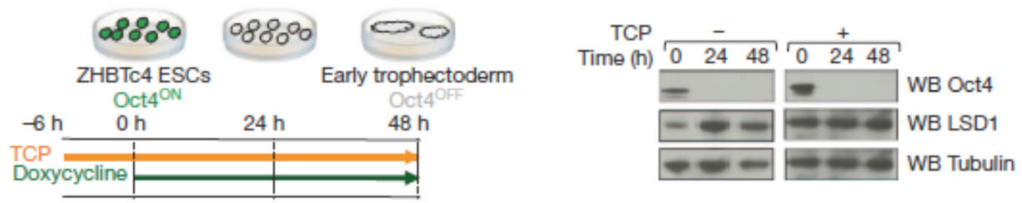

c
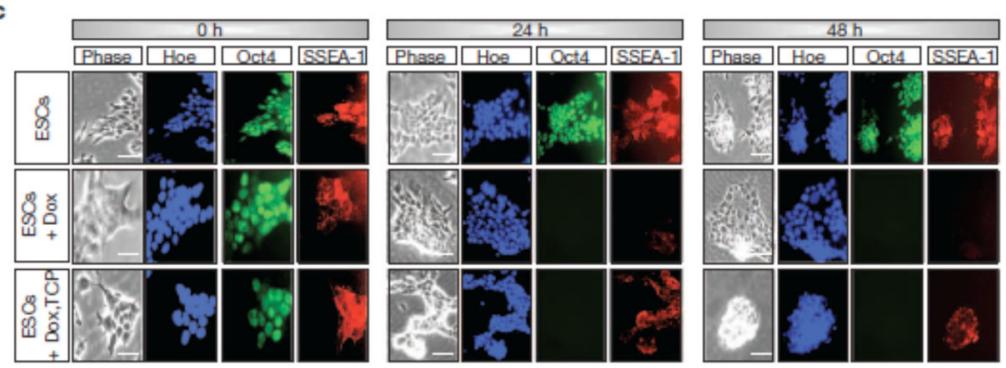

d

ESC genes

Trophectoderm genes
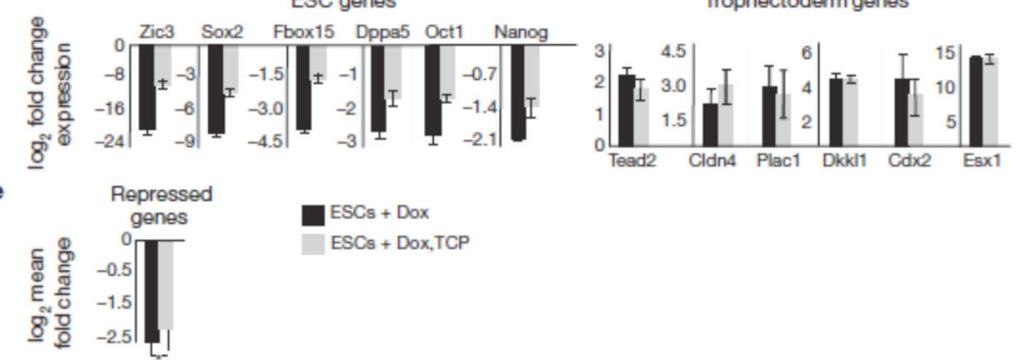

Figure 2. LSD1 inhibition results in incomplete silencing of ESC genes during differentiation a, Schematic representation of trophectoderm differentiation assay using the doxycyclineinducible Oct4 shutdown murine ESC line ZHBTc4. Treatment with doxycycline for $48 \mathrm{~h}$ leads to depletion of Oct4 and early trophectoderm specification. Cells were treated with dimethylsulphoxide (DMSO; control) or the LSD1 inhibitor TCP for $6 \mathrm{~h}$ before $2 \mu \mathrm{g} \mathrm{ml}$ doxycycline was added for a further 24 or 48 h. b. Treatment of ZHBTc4 ESCs with doxycycline leads to loss of Oct4 proteins. Oct4 and LSD1 protein levels in nuclear extracts determined by western blotting (WB) before and after treatment of ZHBTc4 ESCs with $2 \mu \mathrm{g}$ $\mathrm{ml}^{-1}$ doxycycline. Tubulin served as loading control. c, Doxcycline (Dox)-treated cells treated with TCP maintained SSEA-1 cell surface marker expression. Cells were stained for DNA (Hoechst; Hoe), Oct4 and SSEA-1. Scale bar, $100 \mu \mathrm{m}$. d, Expression of selected ESC and trophectodermal genes $48 \mathrm{~h}$ after Oct4 depletion in dimethylsulphoxide-treated and TCP-treated cells (black and grey bars, respectively). Treatment with TCP partly relieved repression of ESC genes but did not affect upregulation of trophectodermal genes. Error bars show s.d. from biological replicates. e, Genes neighbouring LSD1-occupied enhancers are less downregulated during ESC differentiation after TCP treatment. Shown is the mean fold change in expression of the 630 downregulated (at least 1.25 -fold; $P<0.01$ ) genes nearest LSD1-occupied enhancers (Fig. 1c) during differentiation of TCP-treated and untreated control cells. Alleviation of repression is significantly higher (asterisk, $P<0.005$ ) for LSD1 enhancer-bound repressed genes than for all repressed genes. 
a
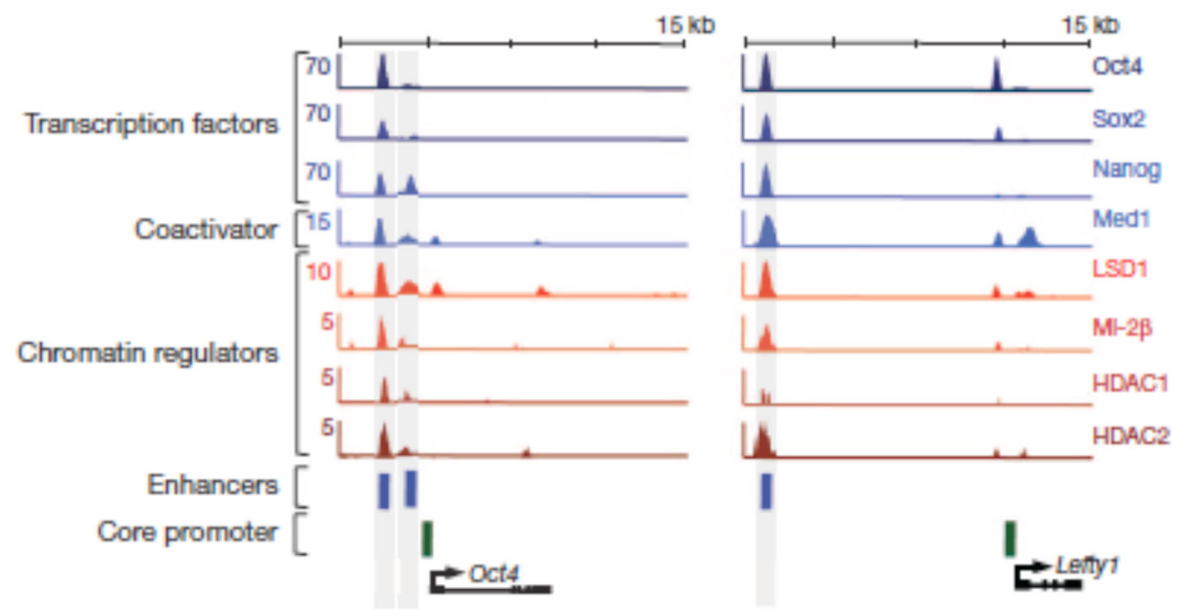

b

C

d
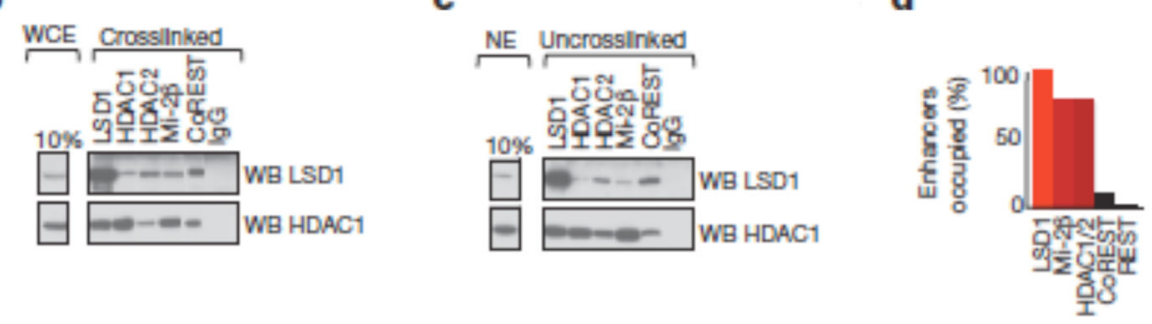

Figure 3. LSD1 is associated with a NuRD complex at active enhancers in ESCs

a, NuRD components occupy enhancers and core promoter regions of actively transcribed genes. Shown are ChIP-Seq binding profiles (reads per million) for transcription factors (Oct4, Sox2, Nanog), coactivator (Med1) and chromatin regulators (LSD1, Mi-2 $\beta$, HDAC1, HDAC2), at the Oct4 (Pou5f1) and Leftyl loci in ESCs, with the $y$-axis floor set to 1. Gene models and previously described enhancer regions ${ }^{27-29}$ are depicted below the binding profiles. b, LSD1 is associated with NuRD components Mi-2 $\beta$, HDAC1 and HDAC2, as well as with CoREST. LSD1 and HDAC1 are detected by western blotting (WB) after immunoprecipitation of crosslinked whole cell extract (WCE) with anti-LSD1, anti-HDAC1, anti-HDAC2, anti-Mi-2 $\beta$ or anti-CoREST antibodies. IgG is shown as a control. c, LSD1 and HDAC1 are detected by western blotting after immunoprecipitation of uncrosslinked nuclear extracts (NE) using anti-LSD1, anti-HDAC1, anti-HDAC2, anti-Mi-2 $\beta$ or antiCoREST antibodies. IgG is shown as a control. d, The occupancy of enhancers by NuRD proteins (Mi-2 $\beta$, HDAC1 and HDAC2) is significantly greater than the occupancy by CoREST or REST $\left(P<10^{-9}\right)$. The height of the bars represents the percentage of the 3,838 enhancers co-occupied by LSD1, NuRD proteins (Mi-2 $\beta$, and either HDAC1 or HDAC2), CoREST and REST. 
a

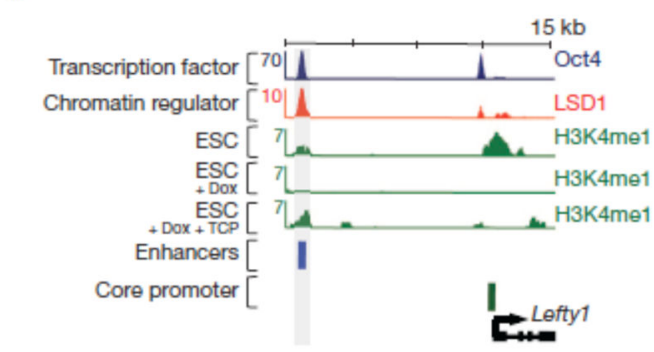

b

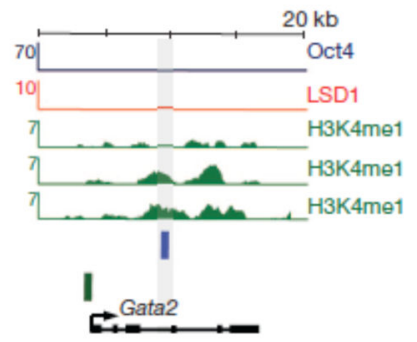

d 1,722 LSD1-

c

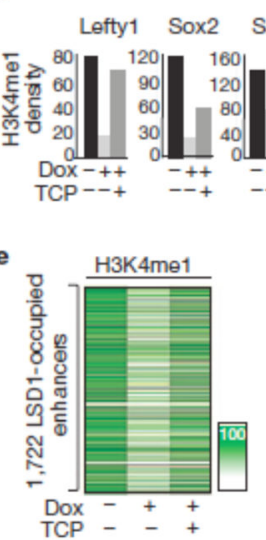
occupied enhancers

Figure 4. LSD1 is required for H3K4me1 removal at ESC enhancers

a, H3K4me1 levels are decreased at LSD1-occupied enhancers during ESC differentiation, and this effect is partly blocked on treatment with TCP. Dox, doxycycline. b, Treatment with TCP does not affect the increase in $\mathrm{H} 3 \mathrm{~K} 4 \mathrm{me} 1$ levels at trophectodermal genes during differentiation. Shown are ChIP-Seq binding profiles (reads per million) for Oct4 and LSD1 at the Lefty1 and Gata2 loci in ESCs. Below these profiles, histone H3K4me1 levels are shown for ZHBTc4 control ESCs, cells treated with doxycycline for $48 \mathrm{~h}$ to repress Oct 4 and induce differentiation (ESCs + Dox), and ESCs treated with doxycycline and TCP $($ ESCs + Dox + TCP). For appropriate normalization, ChIP-Seq data for histone H3K4me1 is shown as rank normalized reads per million with the $y$-axis floor set to 1 (Supplementary Information). Gene models and previously described enhancer regions ${ }^{29,30}$ are depicted below the binding profiles. c, Sum of the normalized H3K4me1 density \pm 250 nucleotides surrounding LSD1-occupied enhancer regions before and during trophectoderm differentiation in the presence or absence of TCP. The associated genes were identified on the basis of their proximity to the LSD1-occupied enhancers. d, Sum of the normalized H3K4me1 density \pm 250 nucleotides surrounding 1,722 LSD1-occupied enhancers before and during differentiation in the presence or absence of TCP. Of the 2,755 LSD1-occupied enhancers with decreased levels of $\mathrm{H} 3 \mathrm{~K} 4 \mathrm{me} 1$ on differentiation, $63 \%(1,722)$ had higher H3K4me1 levels after TCP treatment $\left(P<10^{-16}\right)$. e, Heat map displaying the sum of the normalized $\mathrm{H} 3 \mathrm{~K} 4 \mathrm{me} 1$ density \pm 250 nucleotides surrounding the 1,722 LSD1-occupied enhancers that retained $\mathrm{H} 3 \mathrm{~K} 4 \mathrm{me} 1$ in TCP-treated ESCs compared with untreated control differentiating ESCs. Colour scale indicates ChIP-Seq signal in normalized reads per million. 